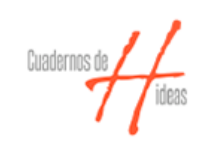

Cuadernos de $\mathrm{H}$ ideas

ISSN: 2313-9048

cuadernosdehideas@perio.unlp.edu.ar

Universidad Nacional de La Plata

Argentina

\title{
Aproximación al análisis organizacional desde la perspectiva de Norbert Elías
}

\author{
Vargas-Hernández, José G. \\ Aproximación al análisis organizacional desde la perspectiva de Norbert Elías \\ Cuadernos de H ideas, vol. 12, núm. 12, 2018 \\ Universidad Nacional de La Plata, Argentina \\ DOI: https://doi.org/10.24215/23139048e015
}

Esta obra está bajo una Licencia Creative Commons Atribución-NoComercial-SinDerivar 4.0 Internacional. 
Artículos

\title{
Aproximación al análisis organizacional desde la perspectiva de Norbert Elías
}

\author{
Approach to organizational analysis from the perspective of \\ Norbert Elías \\ José G. Vargas-Hernández jvargas2006@gmail.com \\ Universidad de Guadalajara, México
}

Cuadernos de $\mathrm{H}$ ideas, vol. 12, núm. 12, 2018

Universidad Nacional de La Plata, Argentina

Recepción: 18 Abril 2018

Aprobación: 10 Julio 2018

DOI: https://

doi.org/10.24215/23139048e015

\section{BY-NC-ND}

Resumen: Este trabajo pretende presentar algunas reflexiones en aproximación al análisis organizacional desde la perspectiva del pensamiento de Nobert Elias. La principal motivación emerge del hueco existente en la literatura teórico-metodológica y empírica que fundamente al análisis organizacional desde los promisorios supuestos de la sociología figuracional centrado en un modelo de la evolución de los procesos psicogenéticos y psicosociales en las relaciones interpersonales de la realidad social, para fecundar las diferentes teorías de las organizaciones y explicar las transformaciones sociales. Este análisis se desprende a partir de las reflexiones de las obras Procesos de Civilización y La Soledad de los Moribundos de Norbert Elias. Se avanza la conclusión de que las formas de organización del poder en las organizaciones se sustentan en el grado de desarrollo evolutivo psicogenético y psicosocial de los individuos, en sus interrelaciones y conflictos entre sí y con las prácticas sociales en su contexto específico, para alcanzar determinados niveles de desarrollo que van de las sociedades con organizaciones e instituciones en barbarie a sociedades civilizadas en donde las relaciones de poder entre los individuos, las organizaciones y el Estado se fundamentan en el pleno ejercicio de los derechos humanos, económicos, civiles, políticos y sociales.

Palabras clave: Análisis organizacional, Norbert Elias, organizaciones civilizadas, Sociología configuracional.

Abstract: This paper intends to present some reflections in an approach to the organizational analysis from the perspective of the thought of Nobert Elias. The main motivation emerges from the existing gap in theoretical-methodological and empirical literature that bases organizational analysis from the promising assumptions of figurational sociology centered on a model of the evolution of psychogenetic and psychosocial processes in the interpersonal relationships of social reality, to fertilize the different theories of organizations and explain social transformations. This analysis is derived from the reflections of the works "The civilizing process" and "The Solitude of the Dying" of Norbert Elias. It is concluded that the forms of organization of power in organizations are based on the degree of evolutionary psychogenetic and psychosocial development of individuals, their interrelationships and conflicts with each other and with social practices in their specific context, to reach certain levels of development ranging from societies with organizations and institutions in barbarism to civilized societies where the power relations between individuals, organizations and the State are based on the full exercise of human, economic, civil, political and social rights .

Keywords: Organizational analysis, Norbert Elias, civilized organizations, configurational sociology.

\section{Introducción}

Tanto los individuos como las organizaciones desempeñan un papel central en los procesos de civilización con una orientación fuerte a la 
inclusión, la interculturalidad y la pluralidad de todas las expresiones. El proceso de civilización organizacional atraviesa por los procesos complejos y contradictorios de modernización y posmodernidad que debe llevar a una liberación progresiva de los individuos de ataduras organizacionales e institucionales. La pertenencia de un individuo a instituciones fuertes como una clase social o a una etnia lo sometía a las reglas formales e informales prescritas de lo que tenían que hacer o no hacer. En la sociología de las organizaciones, el individuo autónomo y libre, civilizado, que participa en las organizaciones pero que tiene la capacidad de tomar decisiones.

Es en este sentido que se afirma que el desarrollo de la democracia como una forma de organización social tiene la capacidad para influir en la organización de la economía como resultado de las prácticas sociales en las interrelaciones del ejercicio del monopolio del uso de la fuerza, el poder y los derechos sociales en consistencia con la concepción del ser humano.

La lectura breve a la obra El proceso de civilización de Norbert Elias (1) ha servido de inspiración para escribir estas reflexiones en torno al impacto que tiene en los estudios organizacionales,(2) pero sobre todo a la concepción de una idea general de las organizaciones civilizadas. En la actualidad, son tiempos en que el proceso de mercantilización de lo humano constituye ya un fenómeno alarmante con serias implicaciones éticas que se configuran en la categoría de virtud en las sociedades civilizadas en el nivel macro y en lo particular y específico en las organizaciones civilizadas a nivel de análisis micro.

Hay en este intento por cubrir un hueco en la literatura la osadía de intentar hacer algunas reflexiones que permitan interesar en el descuidado campo que hasta ahora han tenido los escritos sociológicos de Elias en los análisis organizacionales y su débil impacto a diferencia de otras disciplinas sociológicas. La obra de Norbert Elias trasciende de la sociología a campos afines y por lo tanto puede influir en los estudios organizacionales. Este análisis pretende demostrar cómo algunas de las ideas pueden contribuir a fertilizar los estudios organizacionales. La sociología figuracional de Norbert Elias trasciende su propia disciplina para abordar el análisis de la sociología de las organizaciones, a pesar de que el concepto mismo es controversial y cuente con reconocimientos institucionales de grupos científicos, entre los que cuentan el grupo de Sociología Comparativa desde 2006.

\section{El análisis figuracional}

En el análisis figuracional, el desarrollo de las organizaciones en una perspectiva genética no se presenta en crecimiento cuantitativo sino en niveles de organización internos de las unidades vivientes en la realidad. Sin embargo, se ha retornado a la ilusión de consolidar el prestigio científico a través de la cuantificación del desarrollo de las organizaciones en términos de cambios en la organización de las estructuras sociopsíquicas. 
El enfoque de la sociología figuracional plantea el análisis del desarrollo organizacional en términos de un proceso genético de estadios sucesivos caracterizados por características de organización psíquica como una metodología para resolver los problemas de investigación. Para el caso del análisis organizacional, la genética implica la psicología del desarrollo a través de niveles de organización que surgen, se transforman y se suceden unos a otros con cualidades que se constituyen en procesos cambiantes de las estructuras de organización que son regidas por el concepto de tiempo.

Algunos de los antecedentes del análisis organizacional bajo el enfoque de la sociología figuracional se encuentran en Stacey (3), quien sintetiza la propuesta de Elias y la teoría de sistemas de Nicklas Luhman, en el análisis organizacional, sobre todo en los deportes (4). Iterson y Mastenbroek (5) y Olie e Iterson (6) muestran los estudios sobre la organización informal y la diversidad en las organizaciones para analizar las dinámicas institucionales.

Las organizaciones humanas se hacen en función de la evolución psicogenética y psicosocial de los individuos que la forman y que influye en su conceptualización, así como de las prácticas dominantes en una sociedad. Los fundamentos de una sociedad en proceso de civilización son la personalidad humana, la explotación de la tierra y la organización del tiempo.(7) Estas conceptualizaciones de las tres edades se usan para medir la madurez psicosocial de una sociedad en proceso de civilización a través de la transferencia de valores de una etapa a otra en la historia.

Los procesos de evolución psicogenético y psicosocial de los individuos en sus prácticas sociales son las fábricas de los constructos y modelos organizacionales que puede corresponder al concepto de tradiciones de investigación de Laudan (8) y Velasco (9). El discurso organizacional construye modelos y constructos organizacionales que mediante las prácticas organizacionales posibilitan la construcción de organizaciones predictivas. Así, los diferentes tipos de organización de las relaciones psico-sociales se relacionan en forma positiva o negativa con la convivencia pacífica.

El desarrollo psicogénetico y sociogenético de las organizaciones en proceso de civilización, construye modelos, arquetipos y constructos organizacionales ideales a partir de figuraciones en función del contexto y del tiempo dando lugar a la construcción de tradiciones(10) teórico metodológicas y su implementación mediante prácticas sociales, como una expresión progresiva. Gracias a estas prácticas sociales se construye la realidad organizacional de acuerdo a los modelos, constructos y arquetipos organizacionales. Los constructos o modelos organizacionales prescriben lo que debe ser y lo que puede ser, pero no existen leyes deterministas que posibiliten su implementación sin que se requieran las prácticas sociales.

El modelo de organización burocrático como una forma de organización capitalista, es concebido por Max Weber es una construcción conceptual en el deber ser de un tipo ideal y coherente de organizar que tiene utilidad para el análisis de la realidad organizacional. Las organizaciones de la realidad se acercan al modelo conceptual 
puro en forma de modelo analítico o del mundo del ser. Así, el modelo burocrático de organización concebido por Weber es un tipo ideal, una construcción mental y una herramienta conceptual que se utiliza para el análisis de la realidad de las formas organizacionales. Las regulaciones y normas burocráticas eran funcionales y garantizaban la normalización y estandarización de rutinas en un entorno donde los cambios en el contexto son lentos, pero se ejercía un control de sumisión y manipulación.

En el modelo burocrático, la normalización y regulación racionalmente calculada de las actividades realizadas por individuos enajenados, alienados, despersonalizados por las normas, son de tal forma que vuelve previsibles sus acciones y los resultados. La persona en una organización burocrática no es un individuo en sentido estricto, sin autonomía para decidir qué hacer y cómo hacerlo. Esta forma organizacional contribuyó a la programación de funciones y actividades diferenciadas y en serie de los trabajadores que se especializan solo en una pequeña parte de todo el proceso de producción para el logro de incrementos en la productividad. La exigencia de las normas exige un control estricto sobre los comportamientos en una forma dominante.

Las organizaciones pos burocráticas, así denominadas porque han abandonado el modelo burocrático para adoptar estructuras más flexibles y dinámicas, con capacidades de innovación y más adaptadas a las situaciones complejas e inciertas, al entorno y a las contingencias actuales, aunque en muchos de los casos presentan los síntomas de una degradación burocrática.

Modelos conceptuales como el gobierno del pueblo bajo los supuestos de una idea del ser humano y de la posibilidad de construcción de la ciudadanía, que se determinan por la evolución psicogenética y psicosocial de los individuos, se pueden instrumentar como una forma de organización en el modelo empírico de la democracia de la ciudadanía que garantiza los derechos de todos y cuya realidad depende en mucho de las interrelaciones de prácticas sociales. Estas interrelaciones no están exentas de conflictos. La construcción democrática requiere de estrategias organizacionales para fortalecer las relaciones entre los individuos que son parte de la sociedad civil, el Estado y los partidos políticos.

\section{La teoría elisiana de las organizaciones}

La sociología figuracional de Elias se sustenta en un principio de organización de las polaridades en un equilibrio de las tensiones que ayude a identificar los diferentes niveles de estabilidad en las diversas configuraciones de las interrelaciones. Este nivel de estabilidad del equilibrio puede ir desde las situaciones de total inestabilidad en un extremo hasta la total estabilidad en el otro, de tal forma que se identifican los posibles niveles de violencia existentes en el contexto social. El análisis del campo de las interrelaciones vinculadas a la violencia permite determinar los equilibrios en las tensiones. El concepto de figuración 
de Elías comprende las dimensiones interacción, organización y sistema social como elementos fundamentales de la teoría de sistemas.

El enfoque de la sociología figuracional de Elias está influyendo actualmente en algunos estudios sobre la organización institucional y empresarial. Su pensamiento está en una etapa inicial de aplicación en el análisis organizacional (11) pero existen posibilidades optimistas de un crecimiento de la sociología eliasiana en este campo específico y en muchos otros más en el futuro.(12) El concepto de figuración lo usa Elias en un marco diacrónico de las dimensiones de interacción, organización y sistema social para explicar en cualquier fenómeno social, el entramado de interdependencias, relaciones asimétricas de poder y la no disolución de las interdependencias y las relaciones asimétricas de la relación sociogénesis/psicogénesis.

Elias sostiene un punto de vista de la dinámica relacional y subjetiva de la vida social, más que de objetos, Estados, etc. Lo que propone en sí es la persecución de una reificación de ambas, lo relacional de la vida social y las funciones en su carácter procesal.(13) Esto es, la persona no está solamente auto contenida en una unidad, sino que es un elemento de un conjunto de inter relaciones con otros individuos (14) al igual que en la formación de las organizaciones. Las interrelaciones entre las personas son el objeto de los estudios sociológicos, la parte del cambio histórico: Las circunstancias que hace que el cambio no venga de fuera sino de las relaciones entre las personas.

De esta forma, Elias (15) analiza a la sociedad y su dinámica influido por la concepción weberiana de Estado que posee el monopolio del ejercicio de la violencia legítima en sus formas de expresión de doble coacción para hacer uso de la fuerza militar y para recabar los impuestos. El uso social de la fuerza bruta, se sujeta a un fuerte control social a través de la organización del Estado. "Hoy en día, la crueldad, el gusto de matar y torturar a otros, así como el uso social de la fuerza bruta, se sujeta de modo creciente a un fuerte control social mediante la organización del Estado. Todas estas formas de placer son restringidas por amenazas punitivas y gradualmente "refinadas" y expresadas sólo de modo indirecto. Y es sólo durante períodos de agitación social y guerra, o, en este sentido, en territorios coloniales donde el control social es más laxo, en los que irrumpen de forma más directa y abierta, menos contenidos por sentimientos de vergüenza y repulsión”.(16)

Van Iterson, Mastenbroek, Newton, \& Smith (17) analizan la aplicación de las ideas de Norbert Elias (1897-1990) en los estudios organizacionales y administrativos desde una perspectiva histórica de largo plazo. Muchos de los desarrollos temáticos organizacionales que se inspiran en la obra de Elias están en el debate y en la crítica en las teorías de la organización actuales que tratan sobre las emociones, subjetividad, poder, grupos, estructura, agencia, reuniones, humillación, etc. Por ejemplo, los procesos de humillación en las organizaciones adquieren importancia por sus implicaciones en el contexto social en que se dan, por lo que se requiere analizar el marco de referencia 
socio-histórico, las interrelaciones con las jerarquías para el ejercicio del poder.(18)

Elias (19) estudia los procesos de transformación a largo plazo y utiliza los manuales de comportamiento como evidencia documental empírica para develar los cambios del desarrollo psocogenético y sociogenético de las estructuras psíquicas de los comportamientos y las formas de organización social en el proceso de la civilización. Para el análisis de estas tranformaciones, Elias(20) tipifica los controles básicos que determinan el grado de complejidad del desarrollo de la sociedad: el control de las fuerzas de la naturaleza por los humanos, el control de las interrelaciones entre sí a través de la organización social y finalmente el autocontrol de cada uno de los individuos en una sociedad lo que da la categoría de sociedad civilizada. En las organizaciones e instituciones los que gobiernan tienen el control sobre la violencia legal de una sociedad que determina evitar la violencia ilegal del resto de la misma sociedad.

En la obra El proceso de la civilización, Elias (21) hace un recuento histórico sobre la formación del Estado y las maneras cambiantes de la moralidad y el habitus del viejo orden y ancient regime. Por proceso de civilización, Elías (22) entiende un cambio en la conducta y los sentimientos humanos de una forma muy específica y no un producto de la razón humana o el resultado de una planeación a largo plazo, sino de una reconfiguración de las relaciones humanas aparejado con cambios en la estructura y maneras de la personalidad que dan forma al comportamiento y al sentimiento de civilizados. La civilización es un proceso permanente de transformación y cambio de las estructuras psicológicas y de la organización social.

El libro Sobre el mito del proceso de civilización escrito por Hans-Peter Duerr (23) critica los argumentos del libro El Proceso de Civilización escrito por Elias.(24) El principal argumento es que, si bien Elias demostró el proceso de civilización delimitado por las condiciones sociales, terminó con una auto percepción considerando que la conducta humana llegó a ser más civilizada, aproximándose a una ideología colonial con dominio técnico y militar, con superioridad en la estructura.(25) Los argumentos de Duerr se organizan alrededor del argumento de Elias de que la naturaleza de las restricciones en el cuerpo humano se ejercita en disposiciones psíquicas que cambian de forma de ser agencias sociales externas a localizarse dentro de auto restricciones.

No obstante que ha habido transformaciones históricas en que operan las formas de control social y autocontrol, estas tienen poca correspondencia con el cambio en la efectividad de la restricción psicológica y por lo tanto, no significa que anteriormente en el tiempo las personas hayan sido menos controladas socialmente y auto controladas por sí mismas. El argumento de Duerr (26) se explica en el sentido de que en las sociedades tradicionales que estaban estrechamente interconectadas con los miembros de su propio grupo, el control social directo fue inevitable. Las asociaciones entre las personas también significan una falta de ceguera y una mayor libertad relacional.(27) Para 
Elias, las interdependencias que caracterizan las sociedades urbanas e industriales solamente resultan en la demanda de mayor autocontrol.

Las transformaciones organizacionales se determinan en una relación con el tiempo que va de lo pasivo del reloj fisiológico en una organización simple y primitiva a una determinación activa del tiempo en las organizaciones más complejas y diferenciadas del tiempo social que somete al tiempo individual y penetra en todos los ámbitos de la vida humana. En un estudio más detenido sobre la dimensión del tiempo, Gebara y Florczak (28) como físicos -desde una concepción de la físicaanalizan la evolución figuracional de la noción del tiempo propuesta por Elias para proponer una quinta dimensión basada en el fenómeno de la temporalidad.

Estas transformaciones organizacionales tienen efectos consecuentes en el ejercicio de la autoridad y liderazgo, trabajo en equipo, autoridad personalizada, autocontrol, autonomía, respeto a la dignidad, responsabilidad social. La productividad organizacional requiere de las dimensiones humanas en términos de integración, desarrollo humano, identidad personal, respeto a los derechos humanos, la interculturalidad, equidad de género, tolerancia, responsabilidad social, sustentabilidad y medio ambiente, cultura de paz, ética global, etc. Por ejemplo, el análisis que Elias hace sobre la corte social tiene fuertes implicaciones para la cultura organizacional y corporativa, las relaciones del poder y en general para la sociología de las organizaciones.(29)

En esta transformación, la restricción llega a ser más efectiva en tanto que los impulsos y deseos individuales están más subordinados a los requerimientos de las relaciones sociales diferenciadas y complejas de la interdependencia social. El análisis de Duerr usa evidencias históricas y datos etnográficos para demostrar las dimensiones interculturales de sus argumentos. Duerr considera que no hay un punto cero en los procesos de civilización, ningún período histórico y cultural en la humanidad que no haya sido sujeta a alguna forma de regulación social. Ha habido algunos contra las críticas a los argumentos de Duerr.(30)

En este proceso de civilización quedan en el camino muchos pendientes que resolver para que las organizaciones e instituciones evolucionen a donde el modelo conceptual del sistema democrático como principio de organización social pretende llevarnos: procesamiento de conflictos, funciones de las instituciones legales y culturales, regulación de los mercados, políticas sociales de protección, etc. En el trabajo de Elías (31) sobre la soledad de los moribundos focalizado en las sociedades europeas, encuentra una amplia protección contra las acciones violentas debido fundamentalmente a las instituciones y organizaciones específicas de esa sociedad que alcanzaron eficacia en el monopolio de la violencia, así como a las relaciones económicas de producción y distribución, como resultado de una evolución prolongada.

Es en este sentido que el debate sobre las formas organizacionales del mercado es parte del desarrollo psicogenético y psicosocial de los individuos, así como de las interrelaciones de las prácticas sociales. Estos elementos de civilidad en las organizaciones, instituciones y sociedad 
transitan por diversos procesos de formas de organización de los seres humanos.

La formalización conceptual en el nuevo modelo organizacional de las organizaciones civilizadas, las acciones rutinarias y reguladas, las jerarquías fuertes y autoritarias, las estructuras altas de varios niveles, la diferenciación de funciones, etc., constituyen un obstáculo para la expresión innovadora, multifuncional, de identidad autónoma y de auto-control de los individuos. De hecho, Elias modifica su propuesta teórica de una diferenciación social y una concentración en la formación del Estado con efectos de barbarie y brutalidad para considerar los aspectos alternativos de las organizaciones sociales que tienen efectos civilizantes.(32)

\section{Las traducciones concretas de esta teoría}

La caracterización de los estadios del desarrollo de las organizaciones en un enfoque figuracional se inicia con el posicionamiento específico en tiempo y espacio para el análisis de las transformaciones organizacionales de las representaciones en relación a las acciones concretas, las estructuras del pensamiento y las personalidades ligadas a situaciones específicas. Las interdependencias de las funciones organizacionales en un mismo nivel no se entienden si se tratan por separado.

Los modelos, constructos o artefactos organizacionales son de dos tipos: conceptuales y, empíricos y reales. Los conceptuales constituido por ideas producto de la imaginación e inventiva que, a su vez, resultan de la evolución psicogenética y psicosocial del ser humano y los modelos empíricos reales que, aunque no del todo determinados en relación causal del concepto y hecho social, son contingentes y vinculados a las prácticas sociales, a la intencionalidad de la voluntad humana y al poder. Concebido así el desarrollo de las organizaciones implica un direccionamiento evolutivo bajo la tendencia de una genética supra ordenada de estructuras que se suceden en distintas fases constitutivas.

Por lo tanto, primero se tiene un constructo conceptual que luego se materializa a través de las prácticas sociales en un hecho social institucionalizado. Las organizaciones son producto de la intencionalidad de la voluntad humana que se diseñan y construyen en función de un concepto que luego se objetiviza en la realidad. Existe una relación causal entre el concepto y el hecho social, determinista y con vinculaciones a la intencionalidad del poder y a la voluntad humana. Buenos ejemplos son las propuestas organizacionales del fordismo y el toyotismo que derivaron en prácticas organizacionales.(33)

La conceptualización producto de la imaginación e invención que resulta de la evolución psicogenética y psicosocial, y luego la construcción de modelos organizacionales en función de las prácticas sociales, como en una fábrica, se pueden ubicar en perspectivas prescriptivas más que predictivas de las prácticas organizacionales. La racionalidad del capitalismo ha sido terreno fértil para la emergencia de fábricas de modelos y constructos organizacionales más prescriptivos que predictivos 
de las prácticas organizacionales y que no necesariamente representan lo que es, la realidad organizacional sino lo que debiera ser, como el caso de modelos del fordismo organizacional.

Un modelo o constructo que desarrolló una visión de democracia puede resultar en fuertes diferencias en la objetivización a la realidad de las prácticas sociales en función de la racionalidad económica imperante del capitalismo que favorece otras formas organizacionales, con diferencias prescriptivas de políticas en función de las interrelaciones y los conflictos existentes. Al analizar el grado de desarrollo del modelo de organización social basado en la democracia en una realidad de acuerdo a sus prácticas sociales, se puede delimitar los procesos de civilidad organizacional que están confirmándose y que tienen como supuesto el desarrollo evolutivo psicogénetico y psicosocial de los ciudadanos y la estabilidad que resulta de procesamiento adecuado de los conflictos y las interrelaciones de las prácticas sociales mediadas por las instituciones establecidas en contextos específicos.

Los modelos teóricos metodológicos de las organizaciones adquieren su carácter científico a partir de las teorías clásicas, también llamadas tradicionales y modernas a partir de los trabajos del modelo de administración científica con un claro enfoque a procesos y tecnología y que se instrumenta con el fordismo, confrontado por la teoría del humano relacionismo que centra la atención en los análisis de la importancia de las relaciones humanas en las organizaciones. Al mismo tiempo avanza la modelación y la implementación en las prácticas sociales del modelo burocrático - estructural centrado en la ideación de estructuras organizacionales para la instrumentación de la racionalidad en división de funciones y especialización en las tareas.

En la racionalidad científica, la exigencia de la teoría normativa en la construcción de una filosofía organizacional recupera los conceptos de la visión del deber ser para transformar lo que es, los hechos mismos. Filosofía organizacional enuncia lo que debe ser en el campo de las organizaciones, campo más amplio que lo que es, y, por lo tanto, su objeto de análisis son los conceptos que se refieren a nuevos modelos y constructos humanos o ya construidos y desarrollados que se refieren al cómo debe ser una organización y que representan un estado ideal de las cosas cuya función es posibilitar su construcción organizacional.

En una perspectiva normativa, la filosofía organizacional se orienta a dar sustento a la construcción de las teorías científica sociales que expliquen la realidad de los fenómenos, hagan predicciones verificables y que sean propositivas de nuevos modelos y constructos que se materialicen y solucionen problemas específicos de las organizaciones y la sociedad en su conjunto.(34) La teoría normativa organizacional se ocupa del deber ser de las organizaciones mediante propuestas de modelos específicos de organizaciones para realizar los procesos económicos de producción, distribución y consumo.

Los conceptos y los hechos nuevos solo existen en el deber ser organizacional y no en el ser de las organizaciones. Para que los conceptos organizacionales pasen del deber ser al ser tienen que objetivarse, 
incorporar e instrumentar los valores y visiones adoptados y convertirse en hechos reales que deben existir en las organizaciones. En esta perspectiva de las teorías normativas se debe proponer la implementación de los valores y visiones de una filosofía organizacional en modelos de organización económica de la producción, distribución y consumo que propicien mejoras en el diseño y construcción de organizaciones e instituciones.

La realidad social, a diferencia de la realidad natural, es el resultado de una construcción humana que además se construye intencionalmente en sociedad en base a modelos o constructos de conocimientos posibilitadores.(35) De acuerdo a la Teoría de la acción Comunicativa de Habermas,(36) las organizaciones adoptan patrones de acciones mediante diálogos racionales, logrando acuerdos y consensos y, evitando la fuerza y la violencia. Las instituciones, organizaciones, estructuras, hábitos y costumbres de la realidad social -que es objetiva e independiente de los individuos- son el resultado de patrones de acción producto de un juego estructural e intencional de la construcción humana.(37) Desde otra perspectiva, la objetivización de patrones de acciones, comportamientos e identidades se entienden con la vigilancia y el condicionamiento logrado a través de premios y castigos.(38)

El concepto de segmentación ordenada en el campo de las interrelaciones, si bien los elementos son independientes entre sí, se unifican en situaciones de conflicto y oposición bajo rasgos de formas de estructuras organizacionales que explican desde las alianzas en el futbol hasta la configuración de aglomeraciones humanas en las comunidades urbanas para el apoyo y la defensa del equipo de futbol de su preferencia. El deporte profesional y la organización del trabajo en la economía son representaciones y modos de relación legítimas socialmente afines que se desenvuelven en paralelo con las mismas coordenadas culturales que se reproducen mutuamente. (39)

El proceso de empoderamiento es un proceso de civilización que trae consigo nuevas formas organizacionales a los que son económica, política y socialmente destituidos de todo poder real dotándolos de capacidades para el ejercicio de sus derechos y obligaciones ciudadanas. La agenda pública de la democracia supone una forma de organizar el poder en la sociedad a efecto de que los ciudadanos aborden las diferentes opciones que tienen para los asuntos sustantivos y es por lo tanto un componente esencial de la organización civilizada. Esta agenda de organización ciudadana que se define en el campo de la opción debe contener los retos para los individuos, organizaciones y la sociedad.

En un proceso de civilización, el control en los espacios y tiempos determinados de los cuerpos individuales con reglas bien delimitadas (40) permiten el ejercicio del poder y del gobierno. Este proceso evolutivo de la civilización es consecuencia de los procesos de racionalización, normalización y formalización de las reglas de juego de la organización económica que luego se ajustan al campo de las interrelaciones sociales entre los individuos en forma de acuerdos, contratos y convenios. Este 
proceso de civilización evita la violencia y la guerra continua a partir de los valores que son dominantes en cada sociedad.

En las sociedades donde no existe esta evolución de instituciones y organizaciones de una economía no violenta centrada en elementos de legalidad y cálculo, la violencia está presente y se manifiesta en diversas formas. En una investigación sobre el análisis de los tipos de civilidad en función de la raza y la casa de México, si bien han desaparecido de ser visibles en el discurso público persisten veladamente funcionando como los factores relevantes de la organización y la articulación de las relaciones sociales.(41) En el caso de México, las organizaciones e instituciones que promueven la violencia es porque le han perdido el respeto y el miedo al Estado, y, por lo tanto, repercuten en la seguridad y la confianza de la sociedad mexicana en sus instituciones del Estado y de ella misma.

Las organizaciones civilizadas, en este sentido de que se encuentran en proceso de civilización, creadas por la voluntad de los humanos para satisfacer diferentes tipos de sus necesidades específicas, según las principales razones de los individuos para involucrarse en ellas, generan sus propios modelos o constructos y exigen a sus miembros la aceptación y la instrumentación a través de las prácticas sociales. De la misma forma en que la sociedad civilizada constriñe el proceso de civilización y desarrollo de los individuos para ser aceptados por ella, la construcción y transformación de cualquier organización en proceso de civilización requiere de todos sus miembros primeramente la aceptación de su modelo o constructo ideal para pasar a la objetivación de prácticas sociales específicas.

\section{Conclusiones}

Para Elias (42) en su teoría configuracional, la civilización de la sociedad es el resultado de una construcción, de una transformación. La transformación del ser humano en civilizado sobre el ser natural en función de un modelo conceptual ideal, se realiza en función de acciones coercitivas internas y externas. Elias (43) determina las transformaciones de las estructuras de la personalidad de los individuos en su contexto social en un análisis a largo plazo de las formas de coerción de acciones y emociones corporales para hacer realidad un modelo de ser humano civilizado en proceso constructivo de sociogénesis y psicogénesis.

Las formas de organización del poder en las organizaciones se sustentan en el grado de desarrollo evolutivo psicogenético y psicosocial de los individuos, en sus interrelaciones y conflictos entre sí y con las prácticas sociales en su contexto específico, para alcanzar determinados niveles de desarrollo que van de las sociedades con organizaciones e instituciones en barbarie a sociedades civilizadas en donde las relaciones de poder entre los individuos, las organizaciones y el Estado se fundamentan en el pleno ejercicio de los derechos humanos, económicos, civiles, políticos y sociales.

En las organizaciones como en las sociedades civilizadas, el ejercicio del poder no vulnera esos derechos, en una relación objetiva, y que contribuyan a más elevados niveles de civilidad que faculta a los 
ciudadanos a ejercer la mejor opción cuando se trate de tomar decisiones y elegir en cuestiones sustantivas. En una sociedad en la que florecen las organizaciones en la promoción de los valores cívicos incluyentes de los ciudadanos, su acción conjunta aumenta la participación en los procesos de tomas de decisiones y promueve la transparencia.

\section{Notas}

(1) Elías, Norbert. The Civilizing Process: Sociogenetic and Psychogenetic Investigations. Oxford, Blackwell, 2000.

(2) Iterson, A. v. "Norbert Elias's Impact on Organization Studies". En Adler, P. The Oxford Handbook of Sociology and Organization Studies: Classical Foundations, Oxford, Oxford University Press, 2009.

(3)Stacey, R. Organisational identity: the paradox of continuity and change at the same time, Group Analysis, 38-1, pp. 477-495, 2005a; Stacey, R. "Local and global processes in organizational life", en Stacey, R. (ed), Experiencing Emergence in Organizations, Londres, Routledge, 2005b; Stacey, R. The challenge of human interdependence: consequences for thinking about the day to day practice of management in organizations, European Business Review, 19-4, pp. 292-302, 2007; Stacey, R. Strategic Management and Organisational Dynamics, Pearson, 2011; van Iterson, A., Mastenbroek, W. F. G., Newton, T., \& Smith, D. (Eds.). The civilized organization: Norbert Elias and the future of organization studies, Amsterdam, John Benjamins Publishing Company, 2002.

(4) Maguire, J. Global sport, Londres, Routledge, 1999; Maguire, J. Power and Global Sport. Zones of Prestige, Emulation and Resistance, Londres, Routledge, 2005.

(5) Iterson, A. y Mastenbroek, W. The Civilized Organization. Norbert Elias and the future of organization studies, Amsterdam/Philadelphia, Benjamins Publishers, 2002.

(6) Olie, R. e Iterson, A. "Top management teams in their national context", en Cheng, J. L. C. y Hitt, M.A. (eds).Advances in International Management, Greenwich, JAI Press, 2004.

(7) Benlahsene, B. The socio-intellectual foundations of Malek Bennabi's approach to civilization, London, International Institute of Islamic Thought, 2013.

(8) Laudan, L. Progress and Its Problems. Berkeley, Calif., University of California Press, 1977.

(9) Velasco, A. Resurgimiento de la teoria politica en el siglo XX: Filosofia, Historia y Tradición, México UNAM, 1999.

(10) Laudan, 1977, Op. Cit.

(11) Dunning, E.y S. Mennell. Norbert Elias. London, SAGE Publications, 4 volúmenes, 2003.

(12) Guerra Manzo, E. La sociología figuracionista de Norbert Elias: Críticas y contracríticas. Rencuentro. Análisis de Problemas Universitarios № 66, abril, 2013, pp. 80-89. [en linea] Recuperado (16/07/2017) de: http://www.redalyc.org/articulo.oa? id $=34027019009$

(13) Berger, P. and Luckmann, T. The Social Construction of Reality, Harmondsworth, Penguin, 1971.

(14)van Krieken, R. Norbert Elias and Process Sociology. In Ritzer, G. \& Smart, B. The Handbook of Social theory, Sage, 2001, pp. 353\#67. 
(15) Elias, N. On transformations of aggressiveness, Theory and Society, 5-2, 1978, 227-253.

(16) Ibid, p. 230.

(17) Iterson y otros, 2002, Op. Cit.

(18)Smith, N. Organizations and humiliation: Looking beyond Elias. Organization $8(3), 2001,537-560$.

(19) Elias, N. The Society of Individuals, Oxford, Basil Blackwell, 1991.

(20) Elias, 2000, Op. Cit.

(21) Ibid.

(22) Elías, 1998, Op. Cit., p. 49.

(23) Duerr, Hans-P. Der Erotische Leib, Frankfurt a. M., Suhrkamp, 1997; Duerr, HansP. Obszönität und Gewalt, Frankfurt a. M., Suhrkamp, 1993; Duerr, Hans-P. Intimität, Frankfurt a. M., Suhrkamp, 1990; Duerr, Hans-P. Nacktheit und Scham. Frankfurt a. M., Suhrkamp, 1988.

(24) Mennell, Stephen and Goudsblom, Johan. Civilizing processes - myth or reality? A comment on Duerr's critique of Elias. Comparative Studies in Society \& History 39(4), $1997,729-33$.

(25) Duerr, 1993, Op. Cit., p. 12.

(26) Duerr, 1988, Op. Cit., p. 10.

(27) Ibid, p. 11.

(28) Gebara, M.J. F., y Florczak, M. A. Sobre el tiempo: primeras aproximaciones entre el pensamiento físico y el pensamiento sociológico de Norbert Elias. Política y Sociedad, 50 (2), 2013, 543-552. DOI: http://dx.doi.org/10.5209/rev_POSO.2013.v50.n2.40015

(29) Dopson, S. \& Waddington, I. Managing Social Change: A Process\#Sociological Approach to Understanding Organisational Change within the National Health Service, Sociology of Health \& Illness, 18(4), 1996, 525\#550; Newton, T. Power, subjectivity and British industrial and organisational sociology: the relevance of the work of Norbert Elias Sociology, 33(2), 1999, 411\#40; van Krieken, R. Proto\# governmentalization and the historical formation of organizational subjectivity, Economy \& Society, 15(2), 1996, 195\#221.

(30) Elias, N. Was ich unter Zivilisation verstehe: Antwort aif Hans Peter Duerr. Die Zeit 17.6, 1988; Marx, C. Staat und Zivilisation: Zu Hans, 1996.

(31) Elias, N. La Soledad de los moribundos, México, Fondo de Cultura Económica, Primera edición en Alemán en 1982.

(32)van Krieken, R. The barbarism of civilization: cultural genocide and the 'stolen generations, British Journal of Sociology 50(2), 1999, $295 \# 313$.

(33)Galicia Osuna, D. Filosofía administrativa: la construcción de organizaciones desde constructos o artefactos sociales. Trabajo presentado en el XVIII Congreso Internacional de Contaduría, Administración e Informática. Octubre 2-4 del 2013

(34) Ibid.

(35) Berger, P.L. y Luckmann, T. (2003). La construcción social de la realidad, Amorrortu editores, Buenos Aires, 2003.

(36)Habermas, J. Teoría de la acción comunicativa, I y II, Madrid, Taurus, 1999. 
(37) Giddens A. Las nuevas reglas del método sociológico, Amorrortu, Buenos Aires, 1993.

(38) Foucault, M. Vigilary castigar: nacimiento de la prisión, México, Siglo XXI Editores, 1976. González López, H., Marquina Sánchez, I. y Contreras Asturias, C.C. La civilidad de las castas veladas del México actual. Politica y Sociedad, 20(2), 2013, 517-542. DOI http://dx.doi.org/10.5209/rev_POSO.2013.v50.n2.40016

(39) Vinnai, G. El fútbol como ideología, Siglo XXI, Buenos Aires, 1974, p. 32.

(40) Foucault, M. Space, knowledge and power. In P. Rabinow (Ed.). The Foucault reader (pp. 239-256), New York, Pantheon, 1984.

(41) González López, Marquina Sánchez y Contreras Asturias, 2013, Op. Cit.

(42) Elias, N. (1993). El proceso de la Civilización. Investigaciones sociogenéticas y psicogenéticas, Buenos Aires, Fondo de Cultura Económica, 1993.

(43) Ibid.

\section{Enlace alternativo}

http://perio.unlp.edu.ar/ojs/index.php/cps/index (html) 